\title{
Analyse des sécheresses de 1988 à 1990 en France à partir de l'analyse combinée de données satellitaires NOAA-AVHRR et d'un modèle agrométéorologique
}

\author{
D Courault*, P Clastre, JP Guinot, B Seguin \\ INRA, station de bioclimatologie, centre d'Avignon, BP91, F84143 Montfavet, France
}

(Reçu le 20 décembre 1992; accepté le 2 décembre 1993)

\begin{abstract}
Résumé - Les satellites météorologiques ont été largement utilisés ces dernières années pour le suivi de la végétation, principalement en utilisant l'indice de végétation. Les données thermiques apportent une information complémentaire sur les conditions hydriques des cultures. La température de surface est en effet reliée indirectement à l'évapotranspiration. L'objectif de cette étude est de proposer une méthodologie opérationnelle pour estimer l'évapotranspiration à large échelle, en utilisant de façon combinée des images AVHRR composites de $5 \mathrm{j}$ des satellites NOAA et un modèle agrométéorologique Magret. La relation simplifiée basée sur le bilan d'énergie, qui relie de façon linéaire l'évaporation au rayonnement net et à la différence entre la température de surface et celle de l'air, a été utilisée. Les températures de surface sont déduites des mesures satellitaires à l'aide d'une méthode multispectrale ("split-window") qui a été améliorée pour application aux couverts végétaux. La méthode proposée a été appliquée à l'échelle de la France afin de détecter les effets des sécheresses de 1988 à 1990. II est démontré que les différences de températures entre la surface et l'air, cumulées dans le temps sont significatives des stress hydriques subis par les cultures et permettent de distinguer les variations climatiques régionales. L'analyse des cartes d'évaporation permet une évaluation globale des sécheresses en France au cours des dernières années.
\end{abstract}

température de surface / NOAA / évapotranspiration / modèle agrométéorologique

Summary - Analysis of droughts in France from 1988 to 1990 using NOAA-AVHRR data and an agrometeorological model. The study reported here aimed at proposing an operational methodology to estimate actual evaporation on a large scale, using AVHRR images of the NOAA satellite and an agrometeorological model. The method was applied to detect drought effects for 3 yr (1988-1989-1990) over all of France. For each year, 48 cloud-free images were selected on a 5-d basis. Surface temperatures obtained from AVHRR images were combined with the output data of an agrometeorological model using ground data from 20 stations. The simplified relationship, based on an energy budget relating midday surface-air temperature difference to daily evaporation and net radiation was used for this study. A new local splitwindow method was applied to the images in order to get a better accuracy of surface temperature. Analysis of the cumulative surface-air temperature difference revealed a regional climatic effect. Evaporation maps provided a global evaluation of droughts in all of France for the $3 \mathrm{yr}$ studied.

surface temperature/NOAA/evaporation/agrometeorological model/regional scale

\section{INTRODUCTION}

Les sécheresses observées ces dernières années, en particulier en 1989 et 1990 , ont touché sévèrement l'agriculture de certaines

*Correspondance et tirés à part. régions. La cause principale en est la faible pluviométrie. Les cartes des écarts à la normale des hauteurs de précipitations données par Météo-France mettent en évidence l'évolution de la sécheresse de 1988 à 1990. En 1988 , elle ne concernait que la région sud-est; en 1989, elle a touché l'ensemble de la 
France excepté le nord-est; en 1990, elle s'est aggravée sur les régions précédemment affectées, les déficits en pluie atteignant $500 \mathrm{~mm}$ dans le sud-est et $200 \mathrm{~mm}$ dans le sud-ouest et en Bretagne (Guiméra, 1992). Les réserves en eau s'amenuisent sans être rechargées les années suivantes. Les effets sur les cultures dépendent de la sensibilité des variétés (Gate et al, 1992), mais les chutes de rendement sont souvent importantes. En général, ce sont essentiellement les cultures fourragères qui ont souffert de la sécheresse: en Haute-Garonne par exemple, les estimations données par le SCEES (Service central des enquêtes et études statistiques) pour 1990 ont montré qu'il y avait une diminution de rendements en maïs fourrager de $3 \%$ par rapport à l'année 1989 , et de $15 \%$ par rapport à la moyenne 1984-1988. Les réserves en eau du sol étant inférieures aux besoins des plantes, les zones les plus touchées correspondent naturellement aux sols à faible réserve et peu profonds.

Le diagnostic agroclimatique de la sécheresse s'effectue classiquement par le suivi du bilan hydrique des cultures au cours de l'année, permettant de mesurer "les entrées et les sorties d'eau" afin de détecter les périodes de stress et de mieux gérer les besoins en eau des plantes. $\dot{A}$ des échelles locales (de la plante à la parcelle), des expérimentations sont possibles pour suivre précisément les conditions hydriques des cultures (mesures du potentiel foliaire, du débit de sève, des variations du stock d'eau dans le sol...). À l'échelle régionale, il est difficile d'estimer les différentes quantités d'eau qui interviennent dans le cycle hydrologique, que ce soit pour l'évaporation ou pour les précipitations, à cause des problèmes d'échantillonnage. Deux pistes sont possibles: soit interpoler des valeurs mesurées ponctuellement, soit utiliser des modèles simulant les transferts d'eau et de chaleur des surfaces naturelles. Le modèle Earth (Choisnel, 1977), utilisé par MétéoFrance, permet d'établir des cartes d'évaporation régionales à partir des données climatiques du réseau national. Basé sur la résolution du bilan hydrique d'une surface hypothétique de référence (de type prairie), il traduit directement l'influence du climat et permet de suivre l'évolution des réserves en eau des différentes stations météorologiques en France.

Cette approche comporte des limites, comme pour chaque modèle. Un certain nombre d'hypothèses et de simplifications sont po- sées, qu'il convient de vérifier. Les estimations d'évaporation résultant d'interpolation spatiale apparaissent difficiles à valider à large échelle. Une solution consiste à utiliser d'autres modèles à formalismes différents, en comparant par exemple les sorties de modèles hydrologiques aux sorties du modèle Earth. Mais on manque le plus souvent de mesures de contrôle, ou de points de calage, et les interpolations ne sont pas toujours satisfaisantes... En fournissant sur de larges surfaces, et avec une bonne répétitivité temporelle, l'évolution de certains paramètres utilisés dans ces modèles (comme la température de surface ou l'indice de végétation), la télédétection apparaît comme un moyen privilégié d'analyse, profondément complémentaire des modèles.

Notre objectif a été ici de proposer une méthodologie opérationnelle permettant d'estimer l'évapotranspiration régionalement en utilisant des données satellitaires, et de l'intégrer sur plusieurs mois afin de détecter les effets de sécheresse. Nous avons appliqué cette méthode à l'échelle de la France en comparant 3 années, dont une normale, 1988 , et 2 plutôt sèches, 1989 et 1990 .

En télédétection, les informations dans le visible et le proche infra-rouge ont été largement exploitées par l'intermédiaire de l'indice de végétation pour suivre l'état des cultures (mis en relation avec l'indice foliaire, l'estimation de la biomasse, ou les rendements, Tucker (1979), Guérif et al (1988), en particulier à l'aide des satellites tels que Spot ou Landsat qui ont une haute résolution spatiale (taille du pixel de 20 à $30 \mathrm{~m}$ ). Mais la faible répétitivité de ces images (tous les $26 \mathrm{j}$ pour Spot, avec cependant un accès plus fréquent à partir de la capacité de dépointage, ou tous les $16 \mathrm{j}$ pour Landsat, en tenant compte des 2 satellites en service) limite leur utilisation pour un suivi opérationnel.

L'indice de végétation peut être calculé également à partir des données des satellites météorologiques NOAA (National Oceanic and Atmospheric Administration) qui offrent une couverture mondiale quotidienne avec une résolution de $1 \mathrm{~km}$ au sous-point. Ces données sont mises en relation avec la biomasse (Justice et Hiernaux, 1986; Tucker et al, 1984) et souvent utilisées sous forme de synthèses temporelles afin de suivre l'évolution inter-saisonnière ou inter-annuelle de la végétation (Berges et al, 1989). Les études faites à ces 
échelles larges concernaient jusqu'à présent essentiellement les régions arides à subarides où il y avait peu d'informations au sol. Des unités de surveillance par télédétection ont ainsi été mises en place (au Sahel, la station Agrhymet reçoit des images du radiomètre AVHRR (Advanced Very High Resolution Radiometer) des satellites NOAA et les traite sous forme de cartes de végétation). Ces dernières années à la suite des épisodes de sécheresse qui ont affecté sévèrement l'Europe, des études ont été développées visant à approfondir les possibilités d'application opérationnelle des satellites météorologiques pour le suivi agroclimatique des cultures à l'échelle de la CEE (travaux des équipes INRA-LERTSCEMAGREF dans le cadre du projet européen du CCR-Ispra (Centre commun de recherches) (Seguin, 1992), cartes de synthèse d'indice de végétation réalisées par Geosys). Ces satellites paraissent en effet convenir pour un tel suivi car ils présentent une répétitivité élevée. Leur résolution spatiale est faible mais reste bien adaptée à une échelle régionale ou nationale puisque l'on peut avoir une vision globale de la France de façon instantanée. De plus ces capteurs possèdent des bandes spectrales dans l'infrarouge thermique permettant ainsi d'accéder à la température de surface, variable d'équilibre significative qui traduit bien les changements hydriques de la surface.

Les travaux de l'équipe de Jackson à Phœnix (Jackson et al, 1977) ont montré que l'écart de température entre la surface et l'air (Ts-Ta), mesuré en milieu de journée et utilisé sous forme de son cumul (appelé SDD, Stress Degree Day) est un bon indicateur de l'état hydrique de la végétation. Cet indice permet en effet de différencier les parcelles irriguées des parcelles sèches et de suivre les variations des réserves dans le sol (Steinmetz et al, 1989). Cette même équipe a proposé une relation simplifiée qui relie de façon linéaire ces écarts (Ts-Ta) à des valeurs journalières d'évaporation (ET) et de rayonnement net $(\mathrm{Rn})$ exprimées en $\mathrm{mm}$ d'eau par jour, sous la forme suivante:

$$
E T_{j}-R n_{j}=A+B(T s-T a \max )_{i}
$$

Cette relation dite "simplifiée" par rapport aux autres méthodes d'estimation de l'évapotranspiration a été reprise par de nombreuses équipes aussi bien d'un point de vue physique (Seguin et Itier, 1983; Riou et al, 1988; Lagouarde et McAneney, 1992) que pour des ap- plications opérationnelles (estimation de la réserve utile en eau dans la région de la Crau (Vidal et al, 1987), suivi des conditions hydriques au Sahel (Seguin et al, 1989).

Les relations entre la température de surface et l'évaporation ont donné lieu à différents types de modèles (Sœr, 1980; Taconet et al, 1986), parfois plus ou moins dérivés de la relation simplifiée (Carlson et Buffum, 1989) et appliqués à des échelles locales ou régionales.

Récemment, une étude conduite par Seguin et al (1991), portant sur 17 sites en France suivis de 1985 à 1987, a montré qu'il était possible d'exprimer des différences significatives des conditions hydriques entre sites géographiques et entre années, en utilisant des données thermiques Météosat. Les températures de surface obtenues à partir d'un seul canal de ce satellite ne peuvent être corrigées des effets atmosphériques et sont par suite moins précises que celles obtenues par le capteur AVHRR. Le satellite NOAA possède en effet deux bandes spectrales dans le thermique dont la combinaison par la méthode de "split window" (que nous présenterons page 45) permet de corriger une partie des effets atmosphériques. Par ailleurs la résolution spatiale de Météosat $(5 / 8 \mathrm{~km}$ en France) est moins bonne que celle des données AVHRR $(1,1 / 1,1 \mathrm{~km})$.

En nous situant dans le prolongement de l'étude de Seguin et al (1991), nous nous proposons d'améliorer cette première approche en utilisant directement des données AVHRR du satellite NOAA, qui sont plus précises spatialement et radiométriquement que celles de Météosat, pour le suivi agroclimatique des cultures à large échelle.

\section{RAPPEL DES BASES PHYSIQUES DE LA RELATION ENTRE LA TEMPÉRATURE DE SURFACE ET L'ÉVAPORATION}

La température de surface est reliée indirectement à l'évapotranspiration par le biais du bilan d'énergie. Dans le cas d'une surface mince (sol nu ou couvert bas) que l'on considère en équilibre instantané, le bilan d'énergie s'écrit :

$$
R n=L E+H+G
$$

$R n$ est le rayonnement net représentant la somme des différents rayonnements émis ou 
arrivant à la surface dans les courtes et grandes longueurs d'onde. II s'exprime sous la forme suivante:

$$
R n=(1-a) R g+\varepsilon R a t m-\varepsilon s T s^{4}
$$

$\mathrm{Rg}$ est le rayonnement global, dont une partie aRg est réfléchie par la surface (a étant l'albedo); Ratm est le rayonnement atmosphérique; $\varepsilon \sigma T s_{4}$, le rayonnement émis par la surface; $\varepsilon$, l'émissivité de la surface.

$G$ est le flux de conduction dans le sol, il peut être négligé à l'échelle journalière pour des surfaces couvertes de végétation. La végétation forme, en effet, un écran thermique limitant l'échauffement et le refroidissement du sol. En revanche pour des surfaces de sol nu ou partiellement couvertes de végétation, le flux $G$ n'est pas négligeable (Chanzy, 1991).

$H$ est le flux de chaleur sensible; il s'écrit

$$
H=\rho c_{p} h_{h}(T s-T a)
$$

$H$ dépend de l'écart de température (TsTa) et du terme $h_{h}$, coefficient d'échange de chaleur (inverse de la résistance aérodynamique) $\rho$ et $c_{p}$ étant respectivement la masse volumique et la chaleur spécifique de l'air.

En régime neutre, $h_{h}$ s'exprime par:

$$
h_{h}=k U /\left[\operatorname{Ln}\left((Z-d) / Z_{0}\right)\right]^{2}
$$

où $k$ est la constante de Von Karman; $U$, la vitesse du vent; $Z$, la hauteur de référence; $Z_{0}$, la rugosité de surface; $d$, la hauteur de déplacement traduisant l'effet du relèvement du plan de référence par la structure du couvert.

Cette dernière expression doit être complétée pour tenir compte des effets de stabilité atmosphérique. De jour, (Ts $>$ Tair), le régime est généralement instable et une correction doit être introduite, à partir du nombre de Richardson (Ri) ou de la longueur de MoninObukhov (L).

LE est le flux de chaleur latente, équivalent énergétique de l'évapotranspiration,

$$
L E=\frac{\rho C_{p}}{\gamma} h_{v}\left(e_{s}-e_{a}\right)
$$

où $\left(e_{s}-e_{a}\right)$ représente le déficit de vapeur d'eau dans l'atmosphère; $\gamma$, la constante psychrométrique; et $h_{v}$, le coefficient d'échange de la vapeur d'eau. Généralement, on peut admettre que les coefficients d'échange pour la chaleur et la vapeur d'eau sont égaux: $\mathrm{h}=\mathrm{h}_{\mathrm{h}}=\mathrm{h}_{\mathrm{v}}$.

En utilisant le rayonnement net climatique: $R n^{*}=(1-a) R g+R a t m-\epsilon \sigma T^{4}$, on obtient:

$$
L E-\left(R n^{\star}-G\right)=\left(\rho c_{p} h-4 \varepsilon \sigma T^{3}\right)(T s-T a)[7]
$$

L'évaporation apparaît reliée à la température de surface de façon linéaire. On retrouve ainsi une expression semblable à celle de la relation simplifiée proposée par Jackson et al (1977).

Par ailleurs, si l'on considère les travaux d'Itier et Riou (1982) posant pour des journées claires et ensoleillées, l'égalité des rapports flux de chaleur sensible/rayonnement net, entre les valeurs journalières (j) et instantanées (i) : $\mathrm{Hj} / \mathrm{Rnj}=\mathrm{Hi} / \mathrm{Rni}$, on trouve:

$$
B=(R n j / R n i) \rho c_{p} h
$$

Or d'après Seguin et Itier (1983), $\mathrm{Rnj} / \mathrm{Rni}=0,3 \pm 0,03$ pour des valeurs de rayonnement net instantanées $\left(R n_{i}\right)$ au midi solaire pendant des périodes normalement ensoleillées. B est assimilé à un coefficient d'échange et varie donc essentiellement en fonction du vent et de la rugosité de surface.

Les travaux de Riou et al (1988), puis ceux de Lagouarde et Brunet (1991) et dernièrement ceux de Lagouarde et McAneney (1992) montrent les différentes étapes pour formaliser ces coefficients de calage de la relation simplifiée en fonction des caractéristiques de surface.

Les coefficients les plus couramment rencontrés au cours des nombreuses expériences menées sur la Crau sont: $A=1$ et $B=-0,25$ (Seguin et al, 1982). Ils sont différents de ceux trouvés par Jackson et al (1977): $A=0$ et $B=0,64$, ce qui peut s'expliquer par la différence des rugosités des couverts étudiés (en Crau: il s'agissait de couverts herbacés; à Phœnix, de blé) mais également par la présence de conditions d'advection à Phœnix (une parcelle de blé entourée de zones sèches).

\section{Obtention de la température de surface à partir de capteurs satellitaires}

Le capteur AVHRR de NOAA mesure une luminance spectrale qui est reliée à la température de surface par la loi de Planck.

$$
L_{\lambda}=2 h c^{2} / \lambda^{5} \exp (h c / \lambda k T-1)
$$


avec $L_{\lambda}$ : luminance spectrale propre de la surface, $\lambda$ : longueur d'onde, $h$ : constante de Planck, $\mathrm{c}$ : vitesse de la lumière, $\mathrm{k}$ : constante de Boltzmann, $\mathrm{T}$ : température absolue du corps noir.

Sur des bandes spectrales larges, le signal est intégré; la température de surface apparaît à la puissance $4^{\mathrm{e}}$ (loi de Stefan-Bolztman).

La luminance reçue au niveau du capteur dépend à la fois de l'émission propre de la surface (qui se distingue de celle d'un corps noir par son émissivité) et de celle de l'atmosphère.

$$
L_{\lambda}=\tau_{\lambda}\left[\varepsilon B_{\lambda}(T s)+(1-\varepsilon) L a_{\lambda} \downarrow\right]+L a_{\lambda} \uparrow[10]
$$

avec $L a_{\lambda} \downarrow$ : luminance spectrale propre de l'atmosphère reçue par la surface (souvent appelé rayonnement atmosphérique), $\mathrm{La}_{\lambda} \uparrow$ : luminance spectrale propre de l'atmosphère reçue par le capteur, $\varepsilon B_{\lambda}(T s)$ : luminance de la surface, $\epsilon$ : émissivité spectrale de la surface, $\tau$ : coefficient de transmission atmosphérique.

Pour accéder à la température de surface réelle, il est donc nécessaire de connaître l'émissivité de la surface et le rayonnement atmosphérique.

L'émissivité varie suivant le type de surface, l'humidité, l'angle de visée, les bandes spectrales... ( $\varepsilon=0,90$ pour du sable sec, $\varepsilon=0,98$ pour de la végétation dense). Aussi sa mesure ne se fait-elle que très localement (Stoll, 1988). Une erreur relative de $1 \%$ sur l'émissivité peut conduire à une erreur de $0,75^{\circ} \mathrm{K}$ sur la température de surface (Van de Griend et al, 1991).

De même, l'estimation du rayonnement atmosphérique demande des données, soit mesurées au dessus du sol (utilisation dans ce cas des formules proposées par Brutsaert, 1975), soit issues des profils atmosphériques fournis par des radiosondages (utilisation de programmes tel que Lowtran 6, Kneizys et al, 1983). De telles mesures ne peuvent s'appliquer qu'à des études limitées dans le temps et dans l'espace (Ottlé et Vidal-Madjar, 1992). Elles ne sont pas envisageables dans notre cas, concernant l'ensemble de la France sur plusieurs années. Aussi avons nous considéré que l'émissivité est égale à 1. Compte tenu de l'émissivité élevée du couvert végétal et de l'échelle spatiale large considérée conduisant à des pixels mixtes, l'erreur se situe entre $1 \mathrm{~K}$ et $2 \mathrm{~K}$.
L'autre problème pour l'utilisation des données satellitaires est celui de la perturbation par l'atmosphère. Dans l'infrarouge thermique, si le calcul de l'effet atmosphérique par les modèles de transfert radiatif est difficilement effectuable en routine, il existe la possibilité d'utiliser la méthode dite de split-window qui permet d'éliminer une partie des effets atmosphériques lorsque l'on dispose de 2 canaux thermiques comme c'est le cas pour le satellite NOAA (C4: 10-11.5 $\mu \mathrm{m}, \quad$ C5: 11.5$12.5 \mu \mathrm{m})$. La température de surface est alors calculée à partir d'une combinaison linéaire des températures obtenues dans les 2 bandes spectrales du satellite.

$$
T s=a_{0}+a_{1} T_{c 4}+a_{2} T_{c 5}
$$

Différents coefficients ont été proposés plus ou moins bien adaptés aux types de surfaces rencontrées (Deschamps et Phulpin, 1980; Price, 1983). Récemment Becker et Li (1990) ont montré que ces coefficients pouvaient être calculés à partir des émissivités. Ces données n'étant pas facilement mesurables à de larges échelles, Kerr (1991) a proposé une nouvelle méthode semi-empirique que nous avons employée pour notre étude. Deux températures sont calculées par la méthode de split-window avec des coefficients différents, la première étant plus adaptée à des surfaces de sols nus dominant (Tsn) et la seconde à des couverts denses (Tsv). Ces 2 températures sont ensuite combinées linéairement en fonction d'un paramètre $\mathrm{CV}$ proportionnel à l'indice de végétation pour obtenir la température de surface finale (Ts).

$$
\begin{gathered}
\mathrm{Tsn}=3.1+3.2 \mathrm{~T}_{\mathrm{c} 4}-2.2 \mathrm{~T}_{\mathrm{c5}}, \\
\mathrm{TsV}=-2,4+3,6 \mathrm{~T}_{\mathrm{c} 4}-2,6 \mathrm{~T}_{\mathrm{c} 5} \\
\mathrm{CV}=\alpha \mathrm{IV} \text { avec } \alpha=C V_{1}-\mathrm{CV}_{0} / \mathrm{IV}_{\max }-\mathrm{IV}_{\min } \\
\mathrm{Ts}=\mathrm{CVTsV}+(1-\mathrm{CV}) \mathrm{Tsn}
\end{gathered}
$$

( $\alpha=2$ pour des données non corrigées des effets atmosphériques dans le visible et le proche infra-rouge, cas des images en 1988 et en 1989, $\alpha=4 / 3$ pour les données corrigées, images de 1990). La précision au total des Ts ainsi obtenues est estimée à $\pm 1,5 \mathrm{~K}$ (Kerr, 1991) et, compte tenu des surfaces larges que nous avons considérées, ne doit pas être inférieure à $\pm 2 \mathrm{~K}$ pour l'ensemble de notre série de données. Cette précision est évidemment assez faible en valeur absolue pour une analyse ponctuelle, mais s'avère acceptable lorsque l'on considère les valeurs cumulées sur l'année. 


\section{COMBINAISON DES DONNÉES THERMIQUES AVHRR ET D'UN MODĖLE AGROMÉTÉOROLOGIQUE}

La méthode développée pour cartographier l'évapotranspiration à large échelle consiste à combiner un modèle agrométéorologique et des données thermiques satellitaires. Le modèle (que l'on présentera plus en détail page 47) permet de calculer les différents flux de surface dont l'évapotranspiration réelle (ETR) et le rayonnement net $(R n)$ intervenant dans la relation simplifiée [1], à partir de données climatiques de stations météorologiques. Les données AVHRR des satellites NOAA fournissent les températures de surface. La relation simplifiée est ensuite employée en combinant les sorties du modèle et les écarts de températures entre la surface et l'air afin de déterminer les coefficients de calage $A$ et $B$ pour chacune des stations étudiées.

\section{Les stations météorologiques retenues}

Vingt stations ont été choisies sur l'ensemble de la France, pour lesquelles nous disposions de données climatiques journalières en continu sur les 3 années étudiées (fig 1). Ces sites appartiennent au réseau météorologique géré par le STEFCE-INRA (Service technique des facteurs climatiques de l'environnement). IIs ont été sélectionnés de façon à représenter les principales variations climatiques régionales à l'exception des régions de forêts ou

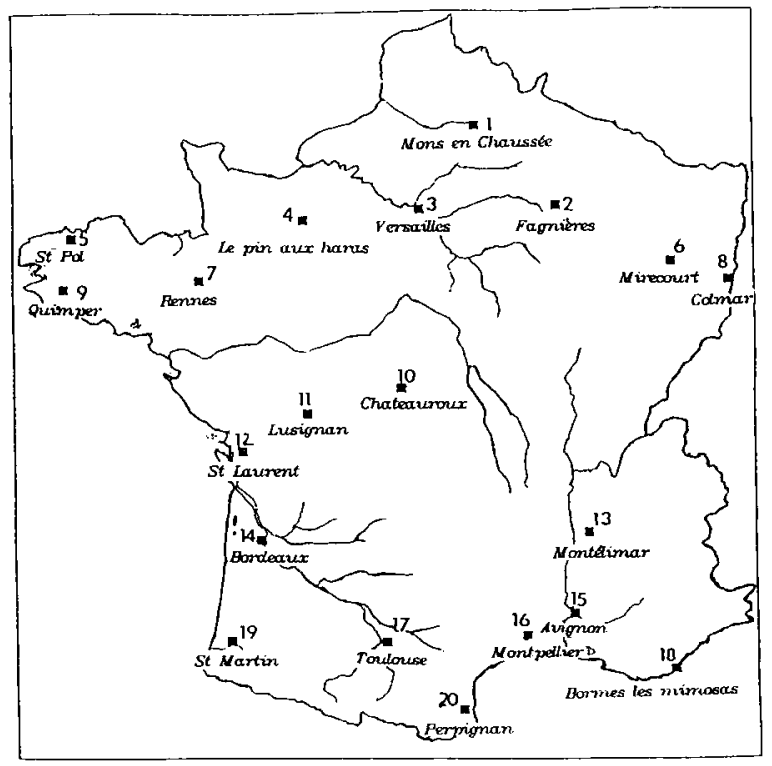

Fig 1. Localisation géographique des sites étudiés sélectionnés à partir du réseau météorologique géré par l'INRA. de montagnes (climat continental pour les sites à l'est et au nord, climat océanique à l'ouest et méditerranéen au sud). Le nombre peut paraître limité, mais il répond à un souci d'opérationnalité, l'augmentation du nombre de sites entraînant des temps de calcul beaucoup plus longs.

\section{Le traitement des images AVHRR}

Les données satellitaires utilisées sont reçues au Centre de météorologie spatiale de MétéoFrance à Lannion. Des images de synthèse sont ensuite réalisées par le CNES à Toulouse en sélectionnant le maximum de l'indice de végétation d'images AVHRR de NOAA prises pendant une période de $5 \mathrm{j}$, durant 8 mois, de mars à octobre (ce qui représente 48 images par an). Cette méthode permet d'éliminer les images nuageuses ou présentant des défauts. Ces images sont rectifiées géométriquement et donc superposables entre elles. L'échantillonnage spatial est d'environ $1 \mathrm{~km}^{2}$. Le repérage de chaque station n'est pas possible à l'échelle du pixel: un léger décalage allant de 1 à 3 pixels peut être observé entre les différentes images. Aussi avonsnous choisi de représenter chaque site par un secteur de $15 \mathrm{~km} / 15 \mathrm{~km}$ (soit 196 pixels), considérant ainsi que les données climatiques ponctuelles sont représentatives d'une petite région géographique. Chaque secteur est caractérisé par la moyenne statistique de ces pixels pour la température de surface.

Un point particulier est à signaler: I'heure de passage du satellite. Une dérive orbitale du satellite NOAA9 qui arrive en fin de vie en 1988 a été constatée, entraînant des écarts qui peuvent aller jusqu'à $2 \mathrm{~h}$ par rapport à l'heure initiale au lancement (Price, 1991). L'heure d'acquisition des images en 1988 varie de $15 \mathrm{~h} 00$ à $17 \mathrm{~h} 00$; la valeur moyenne est à $15 \mathrm{~h} 30$. En 1989, un nouveau satellite est lancé, NOAA11, dont l'heure de passage est proche de $14 \mathrm{~h} 00$. Les températures observées en 1988 ont donc été corrigées de façon à les ramener à $14 \mathrm{~h} 00$, pour pouvoir les comparer aux années suivantes. Les valeurs ont été normalisées en utilisant des relations obtenues à partir de données de terrain acquises par Lagouarde (communication personnelle) avec un radiothermomètre à différentes heures de la journée. La correction effectuée pour les données de 1988 est la suivante

$$
\begin{array}{r}
\text { Ts }_{14 \mathrm{~h}}=(1,03 \pm 0,01)\left(\mathrm{Ts}_{15 \mathrm{~h} 30}-\text { Tair max }\right)+ \\
(3,3 \pm 1,7)+\text { Tair max } r=0,91
\end{array}
$$




\section{Description sommaire du modèle agrométéorologique Magret}

II s'agit d'un modèle couplé bilan d'énergie - bilan hydrique, Magret (Modèle AGrométéorologique d'Évaporation et de Température), adapté par Lagouarde (communication personnelle) à partir du modèle Earth de Choisnel (1977). Le bilan hydrique considère le sol comme un système à 2 réservoirs, dont l'initialisation se fait le 1er janvier d'une année où la réserve d'eau est supposée maximale. Le bilan d'énergie traite les équations classiques des transferts, en prenant en compte les différentes conditions atmosphériques (instabilité thermique...). Les variables d'entrée du modèle sont peu nombreuses: il s'agit des données climatiques journalières disponibles sur le réseau météorologique (pluviométrie, températures de l'air minimale et maximale, température du point de rosée, durée d'insolation, vitesse du vent). Le modèle reconstitue les valeurs horaires des principales variables climatiques. Les paramètres de calage sont de 3 types: géographique $(x, y, z)$, pédoclimatique (texture du sol, réserve utile maximale, température à $1 \mathrm{~m}$ de profondeur, albedo, émissivité, rugosité de surface, rugosité thermique), et biologique (résistance de structure du couvert, indice foliaire). Les principales sorties du modèle sont l'évaporation potentielle (ETP), maximale (ETM) et réelle (ETR), le rayonnement net $(R n)$, la température de surface, la réserve en eau du sol, à un pas de temps journalier.

Les principales modifications apportées par Lagouarde au modèle initial Earth (Choisnel, 1977) concernent la prise en compte séparée de la pluie et de l'évaporation, de l'instabilité atmosphérique et de la rugosité thermique intervenant dans le calcul du coefficient d'échange, l'introduction de l'indice foliaire atténuant le flux de conduction dans le sol. Pour notre étude, les 20 stations ont été assimilées à une prairie. Seules les données climatiques et pédologiques varient.

Compte tenu de ces caractéristiques, ce modèle est adapté à une estimation de l'ETR locale plutôt que régionale. Tel que nous l'avons utilisé, il nous fournit une référence d'ETR de signification essentiellement climatique, permettant de comparer les situations hydriques entre sites et années.

\section{Les différentes étapes de l'analyse des données}

La combinaison des différentes données thermiques, climatiques et des sorties du modèle s'est effectuée en 3 étapes:

i) dans un premier temps, nous avons examiné les écarts (Ts-Ta) et leurs cumuls (SDD) de mars à octobre pour les 20 sites étudiés, afin de détecter quel sont les secteurs ayant subi les stress hydriques les plus importants;

ii) dans un second temps, les valeurs d'ETR et de $R n$, calculées par le modèle pour les 20 sites ont été combinées aux écarts (Ts-Ta) suivant la relation simplifiée, afin d'obtenir des coefficients de calage $A$ et $B$ applicables à l'ensemble de la France. Les coefficients ont été calculés également par station afin d'apprécier la variabilité interannuelle des sites.

La confrontation de données spatiales obtenues pour l'ensemble du territoire (images thermiques) avec des données ponctuelles (Tair sur 20 sites) interpolées pose le problème de la représentativité spatiale de la température de l'air. II faut cependant noter que la température de l'air est plus homogène que la température de surface. Une amélioration pourrait être apportée en utilisant des cartes interpolées obtenues par Météo-France à partir d'un réseau plus dense de stations et de modèles d'interpolation plus adaptés;

iii) enfin la troisième étape a consisté à obtenir des cartes d'évaporation à l'échelle de la France. Des cartes interpolées par krigeage des variables climatiques (Tair max et $\mathrm{Rn}$ ) ont été utilisées, conjointement aux images de synthèse AVHRR afin de détecter les secteurs les plus touchés par les sécheresses.

\section{RÉSULTATS}

\section{Analyse des écarts (Ts-Ta) cumulés de mars à octobre}

Les écarts de températures entre la surface et l'air varient essentiellement en fonction de trois facteurs:

i) la latitude: la différence entre les sites nord et sud est bien marquée (fig 2);

ii) l'occupation de la surface: les secteurs où les sols nus sont dominants (cas de Perpignan) présentent des températures de surface supérieures à celles de secteurs où la cou- 


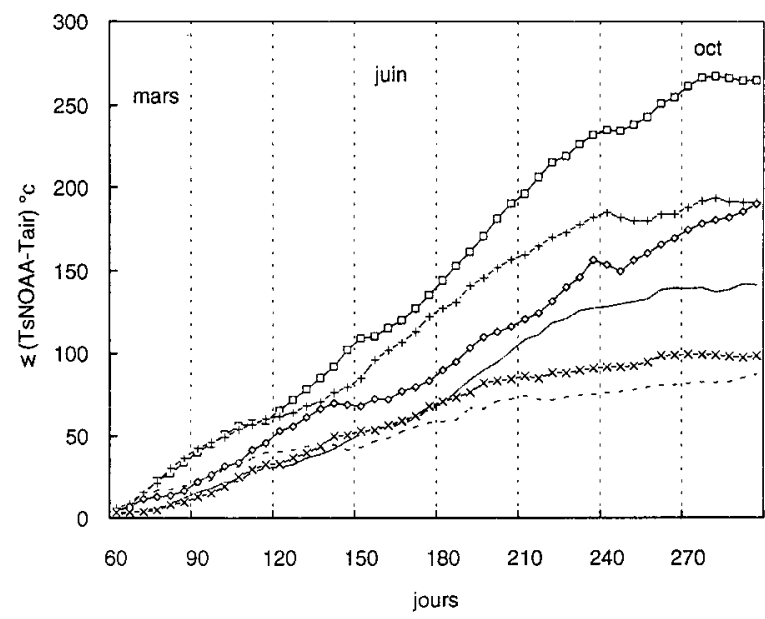

Fig 2. Évolution des écarts cumulés (TSNOAA-Tair max) de quelques stations de mars à octobre 1988. - $\square-$ Perpignan, -- Avignon, - $\diamond-$ Saint-Laurent, -- Rennes, -x-Quimper, -..-- Mirecourt.

verture végétale est dense (tel que Mirecourt) ou marécageux (Saint-Laurent);

iii) la proximité des côtes, où les vents et l'humidité de l'air sont plus forts et ont pour effet d'abaisser les écarts (Ts-Ta), exemple de Quimper (fig 2).

Pour un même site, les variations des écarts de températures observées entre les 3 années sont significatives des déficits hydriques subis (fig $3 a, b$ ). Ces variations sont plus ou moins importantes suivant les régions: la différence entre les années sèches (1989, 1990 ) et l'année plus humide (1988) est nettement plus accentuée dans le secteur sudouest (Toulouse) que dans le secteur d'Avignon. La forte sécheresse de 1989 pour ce secteur $(389 \mathrm{~mm}$ de pluie sur l'année contre $625 \mathrm{~mm}$ en 1988 et $690 \mathrm{~mm}$ en 1990 , avec seulement $16 \mathrm{~mm}$ pour juin-juillet-août contre respectivement $84 \mathrm{~mm}$ en 88 et $75 \mathrm{~mm}$ en 90) n'est pas identifiable, mais on peut penser qu'elle est compensée, au niveau des images satellitaires dans l'infrarouge thermique, par les apports d'irrigation très notables sur une forte part de la surface agricole.

\section{Calcul de l'évapotranspiration}

Les valeurs d'évapotranspiration réelles calculées par le modèle Magret sont, pour la plupart des sites, plus élevées en 1988 qu'en 1989 et 1990 . La différence est surtout marquée pendant les mois d'été à partir du mois de juin où les déficits hydriques sont les plus importants pour les 2 dernières années (fig 4). Les valeurs cumulées de mars à octobre s'étendent de $215 \mathrm{~mm}$ à $545 \mathrm{~mm}$ pour l'ensemble des stations (tableau I). Un gradient dû à la latitude est bien marqué dans certains secteurs comme la vallée du Rhône ou le Sud-Ouest (transect Bordeaux Toulouse Perpignan, fig 5). On constate en effet que l'évaporation réelle diminue des régions les plus humides (Bordeaux) aux régions les plus sèches (Perpignan), tandis que l'évaporation potentielle (traduisant la demande climatique) présente une variation inverse, conformément aux observations de Seguin et al (1991).

\section{Détermination des coefficients de calage de la relation simplifiée}

Les régressions calculées entre les valeurs d'ETR et de Rn cumulées de mars à octobre et les différences de températures ( TS $_{\text {NOAA }^{-}}$ Tair) cumulées (le cumul est réalisé avec une valeur par pentade, voir $p 46$ ) permettent d'obtenir les coefficients $A$ et $B$ de la relation simplifiée (équation 1). Des variations de pente s'observent entre les différents sites (fig 6), et parfois pour un même site entre les différentes années. Ces coefficients $A$ et $B$ sont fortement dépendants de la rugosité de surface et de la vitesse du vent (voir p 44). Ces différences peuvent donc s'expliquer par des variations significatives de l'occupation de surface au niveau de la rugosité de surface (variation du pourcentage de forêts, de cultures, ou de sols nus) et des conditions climatiques (régimes de vent variables d'une région à une autre). Dans la région Midi-Pyrénées par exemple, l'occupation de la surface a changé entre 1989 et 1990: près de $30 \%$ des surfaces cultivées en maïs en 1989 ont été remplacées par du tournesol en 1990 , à cause des faibles réserves du sol, et des craintes de la sécheresse (Rapport Geosys, 1990). Le tournesol demande moins d'eau et présente une surface moins couvrante que le maïs, aussi on peut penser que les temperatures de surfaces et l'indice de végétation peuvent varier légèrement d'une année sur l'autre. La pente $B$ varie peu et reste quasiment constante pour certains sites comme Perpignan où les sols nus sont dominants.

À la suite de ces observations, 2 types d'approches peuvent être proposées pour cartographier l'ETR à l'échelle de la France: 

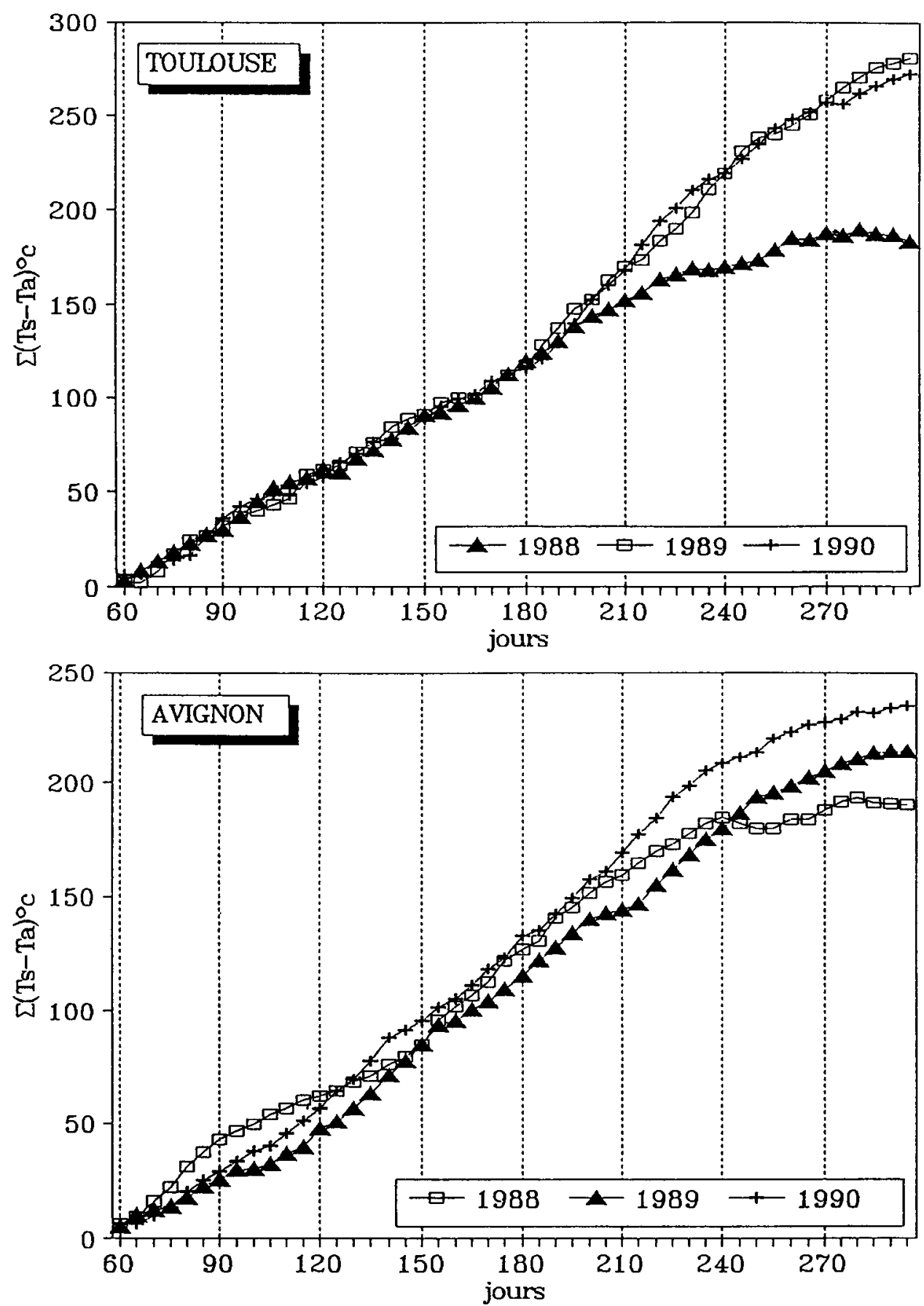

Fig 3. Comparaison des écarts cumulés de mars à octobre (TSNOAA-Tair max) des sites de Toulouse et d'Avignon pour les 3 années 1988, 1989, 1990.

i) soit interpoler par krigeage ou par d'autres méthodes les sorties du modèle (l'ETR);

ii) soit interpoler les données d'entrée (les variables climatiques: $\mathrm{Rn}$, Tair).

La première voie a été explorée par Seguin et al (1991) à partir de données Météosat des années 1985 à 1987 . Une délimitation des aires d'influence des sites a été effectuée en utilisant la méthode des polygones de Thiessen. Les valeurs des coefficients $A$ et $B$ ont ensuite été calculées pour chacune de ces zones. Les valeurs d'ETR obtenues par la relation simplifiée ont ensuite été interpolées à l'échelle de la France. La délimitation des zones d'influence se faisant sur des critères purement géométriques nous a semblé insa- 


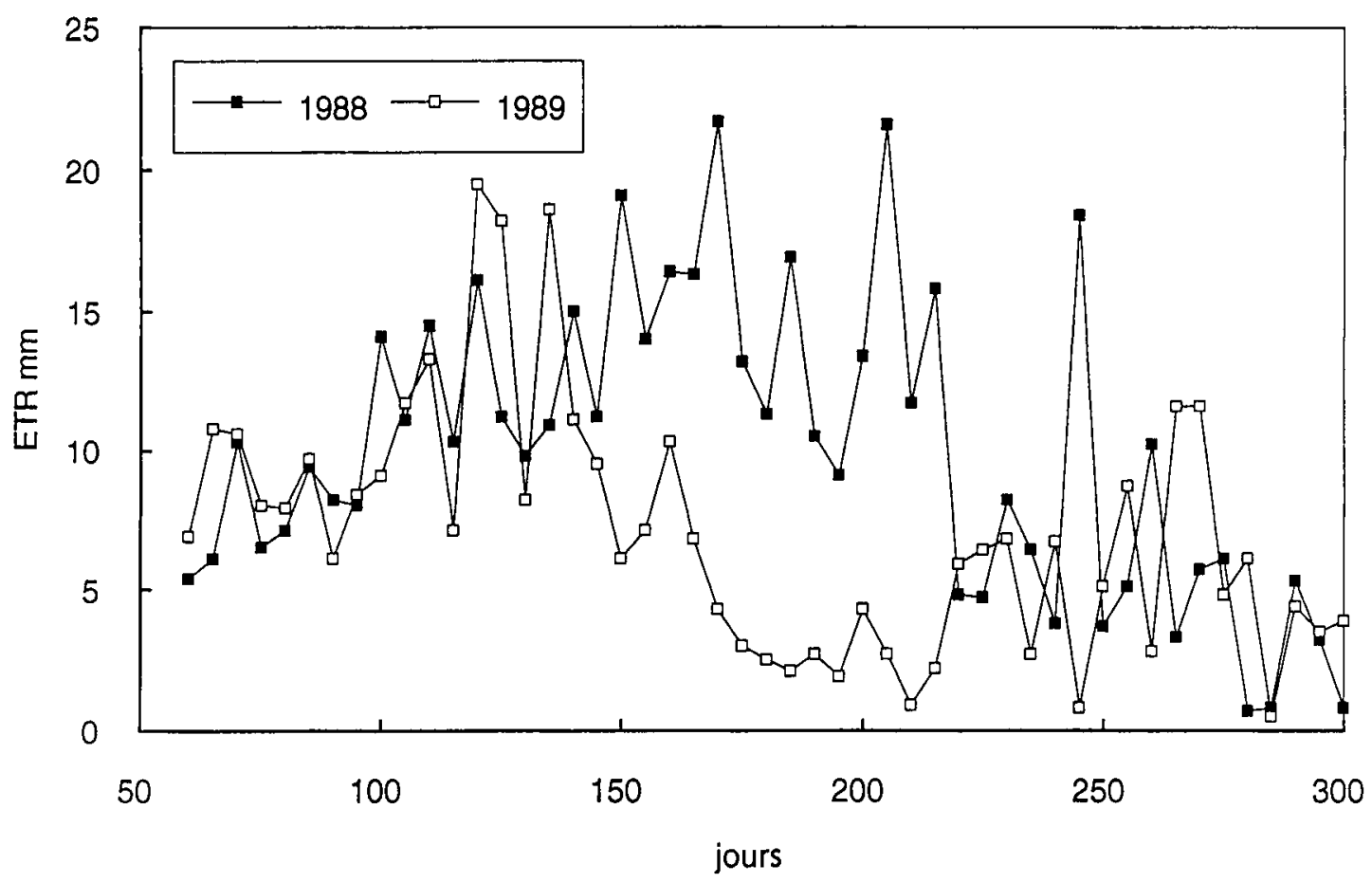

Fig 4. Comparaison des valeurs d'évaporations réelles calculées par Magret pour le site de Toulouse en 1988 et 1989 (valeurs pentadaires).

Tableau I. Caractéristiques moyennes des 20 sites de mars à octobre.

\begin{tabular}{ccccc}
\hline $\begin{array}{c}\text { Pluie } \\
(\mathrm{mm})\end{array}$ & $\begin{array}{c}\text { Tairmax } \\
\left({ }^{\circ} \mathrm{C}\right)\end{array}$ & $\begin{array}{c}\text { TsNOAA } \\
\left({ }^{\circ} \mathrm{C}\right)\end{array}$ & $\begin{array}{c}\text { ETRMagret } \\
(\mathrm{mm})\end{array}$ \\
\hline 1988 & $494 \pm 134$ & $20,4 \pm 2,1$ & $23,3 \pm 2,7$ & $445 \pm 75$ \\
1989 & $350 \pm 105$ & $22,2 \pm 2,0$ & $25,8 \pm 2,7$ & $357 \pm 58$ \\
1990 & $403 \pm 115$ & $21,8 \pm 1,9$ & $26,0 \pm 2,5$ & $391 \pm 73$ \\
\hline
\end{tabular}

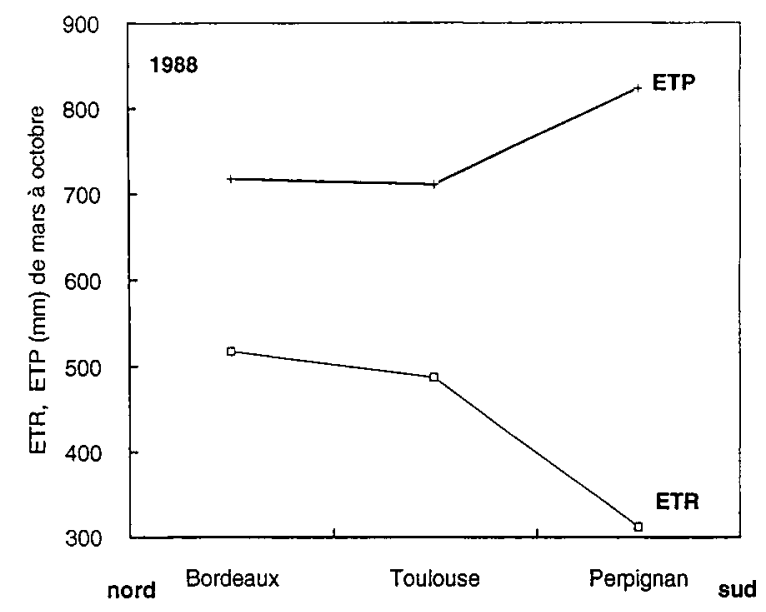

Fig 5. Évolution de l'ETP (Penman) et de l'ETR (Magret) le long d'un transect latitudinal en 1988. tisfaisante, aussi avons-nous choisi la seconde approche qui consiste à interpoler les variables climatiques (Tair et $\mathrm{Rn}$ ). Cette voie présente l'intérêt d'élaborer des cartes intermédiaires ( $\Sigma T s, S D D)$ qui peuvent déjà être interprétées en termes de stress hydriques sur l'ensemble du territoire.

Les coefficients calculés à partir des 20 stations (tableau II) ont servi de coefficients de calage pour la cartographie finale de l'ETR. L'utilisation de coefficients régionaux serait sans doute plus appropriée, mais la sélection des zones concernées posant des problèmes de définition des limites, nous avons préféré conserver une continuité spatiale en choisissant des valeurs uniques pour l'ensemble du territoire. Les erreurs induites sur l'ETR par cette approximation sont de l'ordre de 20 à $30 \%$ (Seguin et al, 1991) suivant les stations. Ces résultats donnent un premier ordre de grandeur relativement raisonnable des différences spatiales d'ETR à l'échelle de la France, qu'il est difficile d'apprécier par d'autres méthodes d'estimation des flux. L'expérience FIFE menée aux USA a fait apparaître des ordres de grandeur comparables, de l'ordre de 20 à $30 \%$ dans l'incertitude d'estimation des flux à l'échelle régionale (Kanemasu et al, 1992). 


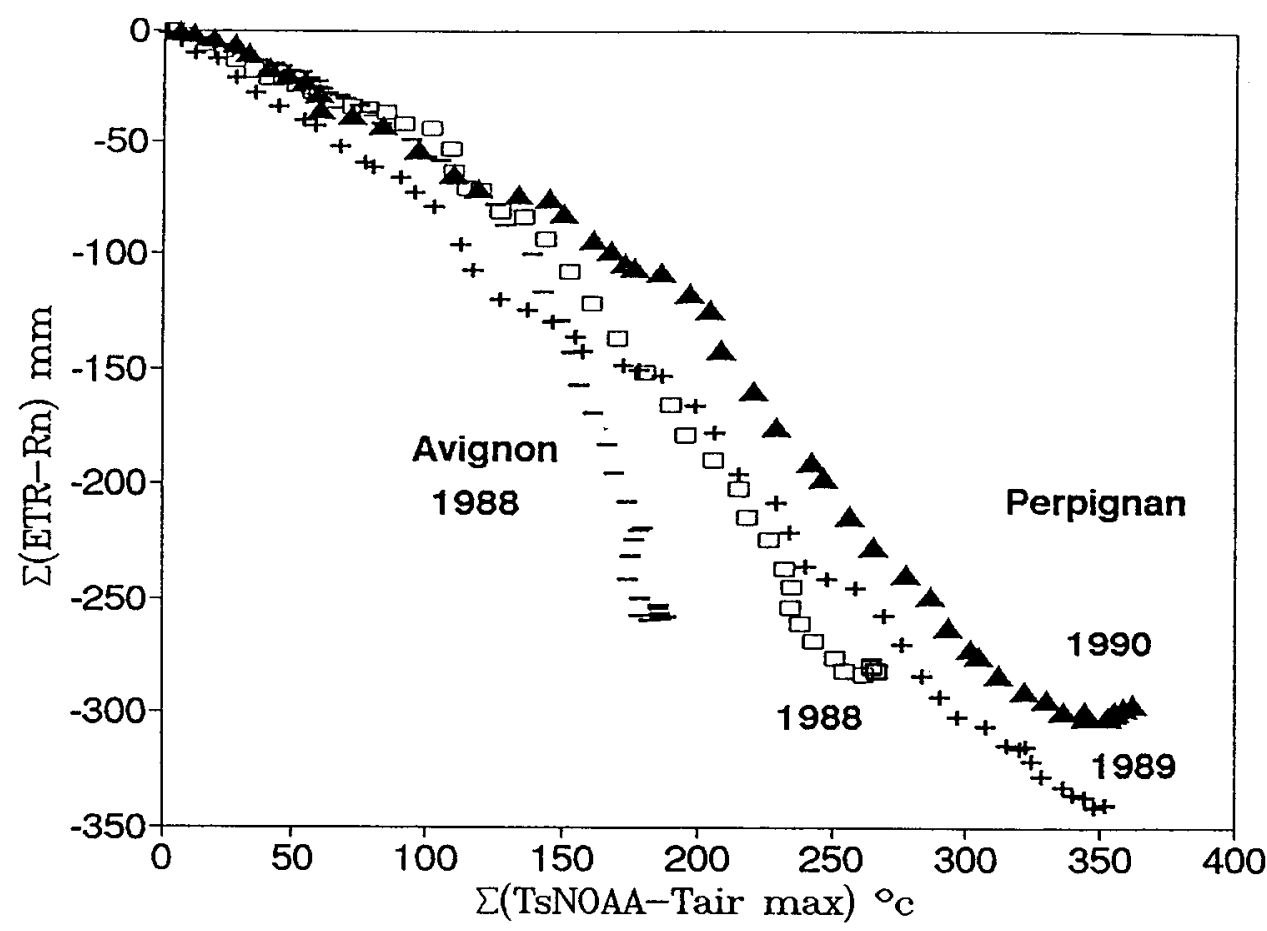

Fig 6. Relation simplifiée obtenue pour 2 sites $\Sigma(E T-R N)=A+B \Sigma(T s-T a)$. Perpignan $\left(B 88=-1,18 r^{2}=0,95, B 89=-1,02\right.$ $\left.r^{2}=0,99, B_{90}=-0,94 r^{2}=0,97\right)$. Avignon $\left(B 88=-1,51, r^{2}=0,89\right)$.

Tableau II. Coefficients de la relation simplifiée obtenus en considérant les 20 sites.

\begin{tabular}{cccc}
\hline & $n A$ & $B$ & $r^{2}$ \\
\hline 1988 & 10,35 & $-1,09$ & 0,72 \\
1989 & $-12,2$ & $-1,19$ & 0,66 \\
1990 & $-1,93$ & $-1,07$ & 0,76 \\
\hline
\end{tabular}

$\Sigma(E T R-R N)=n A+B \Sigma(T s-T a) ; n=48$ pentades de mars à octobre.

\section{Comparaison avec une estimation d'ETR issue d'une approche hydrologique}

Les valeurs d'évaporations réelles ainsi estimées sont à considérer avec précaution et n'ont qu'une valeur relative, car il n'existe pas de méthode de validation à des échelles de temps et d'espace aussi larges. Un moyen peut être envisagé néanmoins à l'échelle régionale, en considérant le bilan hydrologique de bassins versants. En effet, certains modèles spatialisés comme Modcou (Girard et al, 1981) permettent des sorties de cartes d'évaporation (Perrin, 1991) qui peuvent être comparées à nos estimations d'ETR utilisant des données AVHRR. Comme nous ne possédions pas de telles données pour les années étudiées, nous avons testé la formule de Turc (1954) qui s'adapte à des bassins versants pour calculer l'ETR, gardant ainsi une approche hydrologique. L'évaporation est calculée à partir d'une équation faisant intervenir des valeurs moyennes de températures et de pluies cumulées, au cours d'une année, représentatives d'un bassin versant. Nous l'avons adaptée sur une période de 8 mois:

$$
\mathrm{ET}=\frac{\mathrm{P}}{\sqrt{0,9+\mathrm{L}^{2} / \mathrm{P}^{2}}}
$$

avec $L=300+25 t+0,05 t^{3}, \quad P$ : précipitation annuelle $(\mathrm{mm}), \mathrm{t}$ : moyenne annuelle de la température de l'air $\left({ }^{\circ} \mathrm{C}\right)$. Cette relation s'applique si $L^{2} / P^{2}>0,1$ sinon on pose $E T=P$.

La comparaison des valeurs d'ETR calculées par Magret et celles calculées par la formule de Turc montre une bonne concordance (fig 7). Les écarts moyens observés entre ces

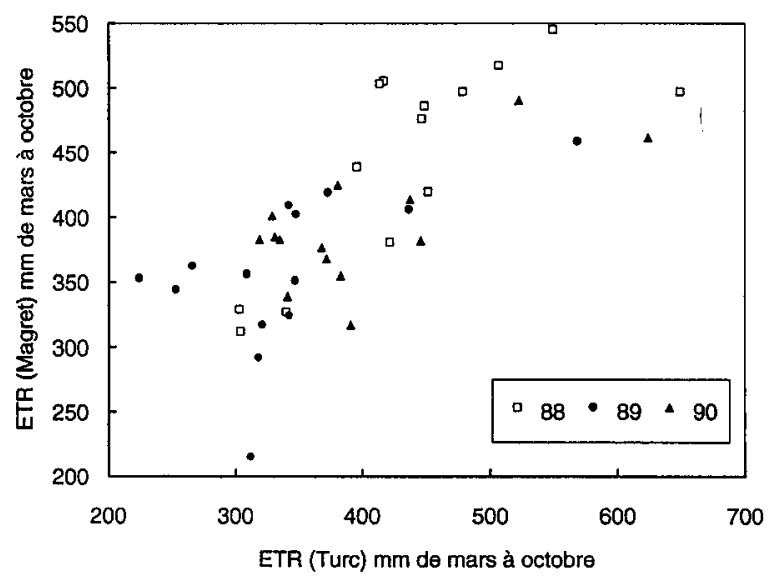

Fig 7. Corrélation entre les valeurs d'ETR cumulées de mars à octobre, calculées d'après la formule de Turc (1954) et celles calculées par le modèle Magret pour les 20 sites étudiés au cours des 3 années 1988, 1989 et 1990. 

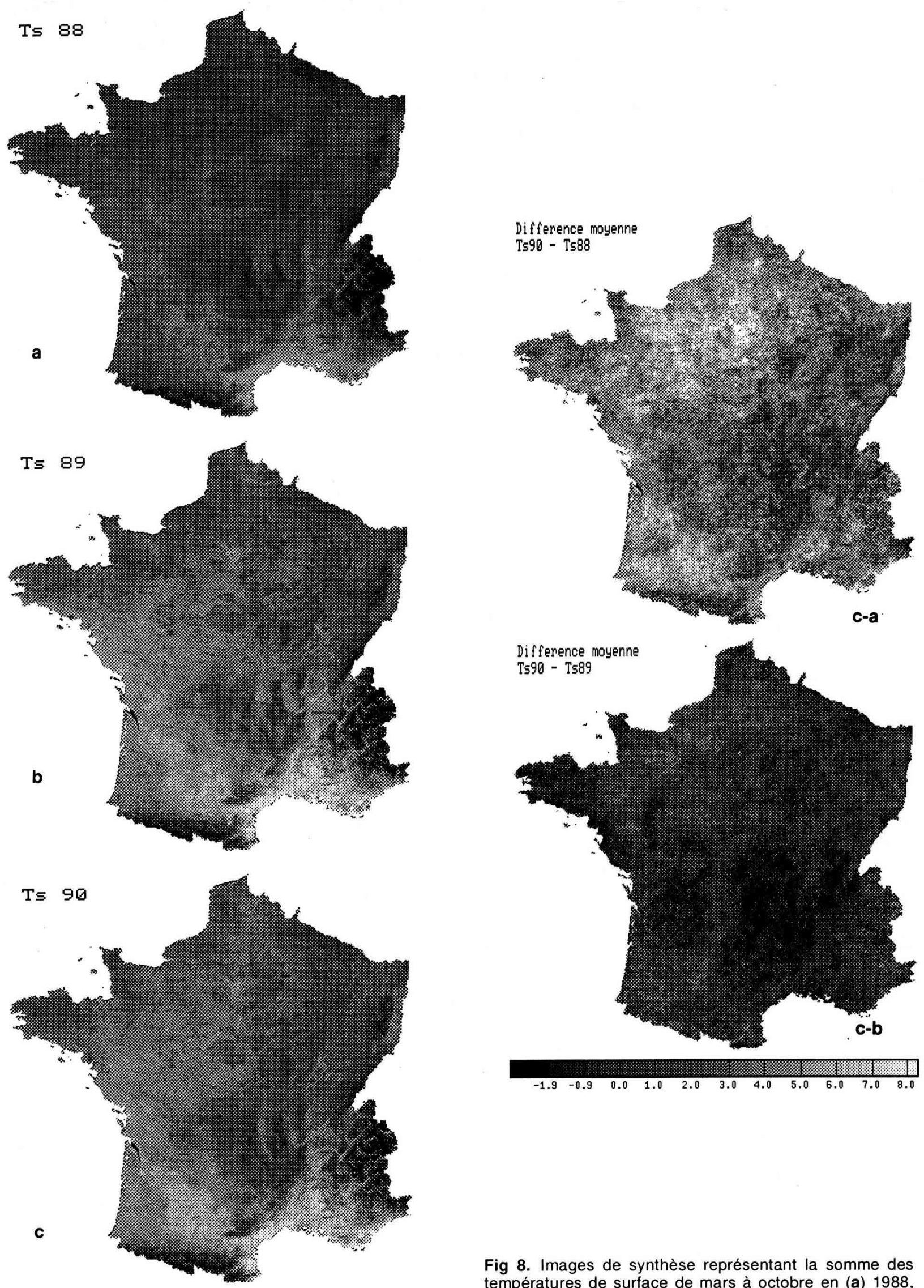

Fig 8. Images de synthèse représentant la somme des températures de surface de mars à octobre en (a) 1988, (b) 1989, (c) - 1990. Différence des températures de surface en 1990 et 1988 (image (c) - (image (a), et entre 1990 et 1989 (c)-(b). 


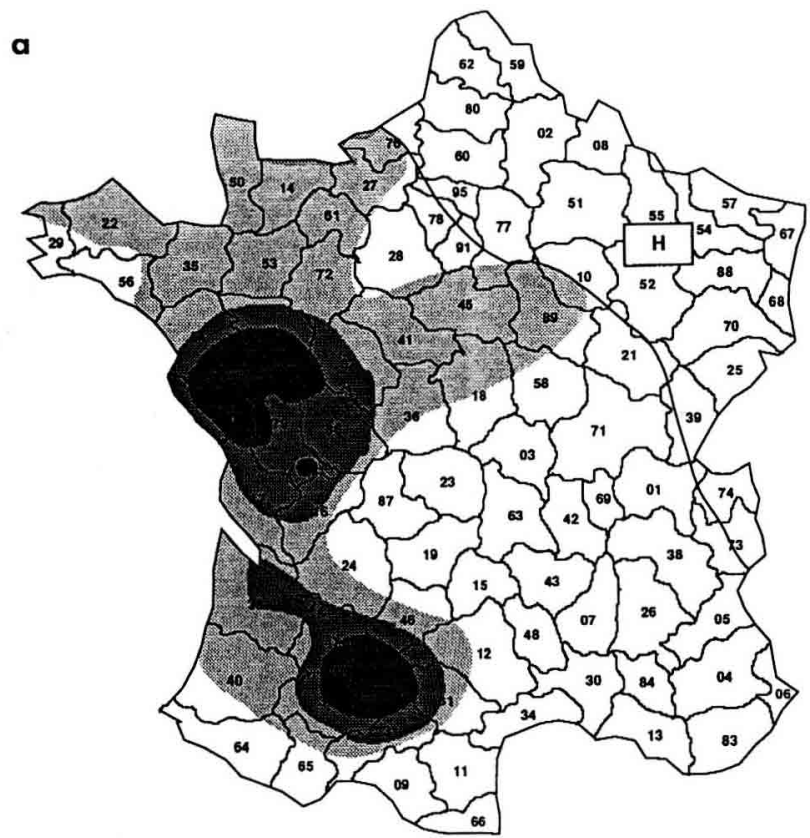

$\mathrm{H}$ Zone hors d'étude

Végétation peu touchée

Végétation moyennement touchée

Végétation fortement touchée

Végétation très touchée

Fig 9a. Carte de synthèse sur la sécheresse en 1990 réalisée à partir de l'indice de végétation calculé sur une série d'images NOAA (in Geosys 1990).

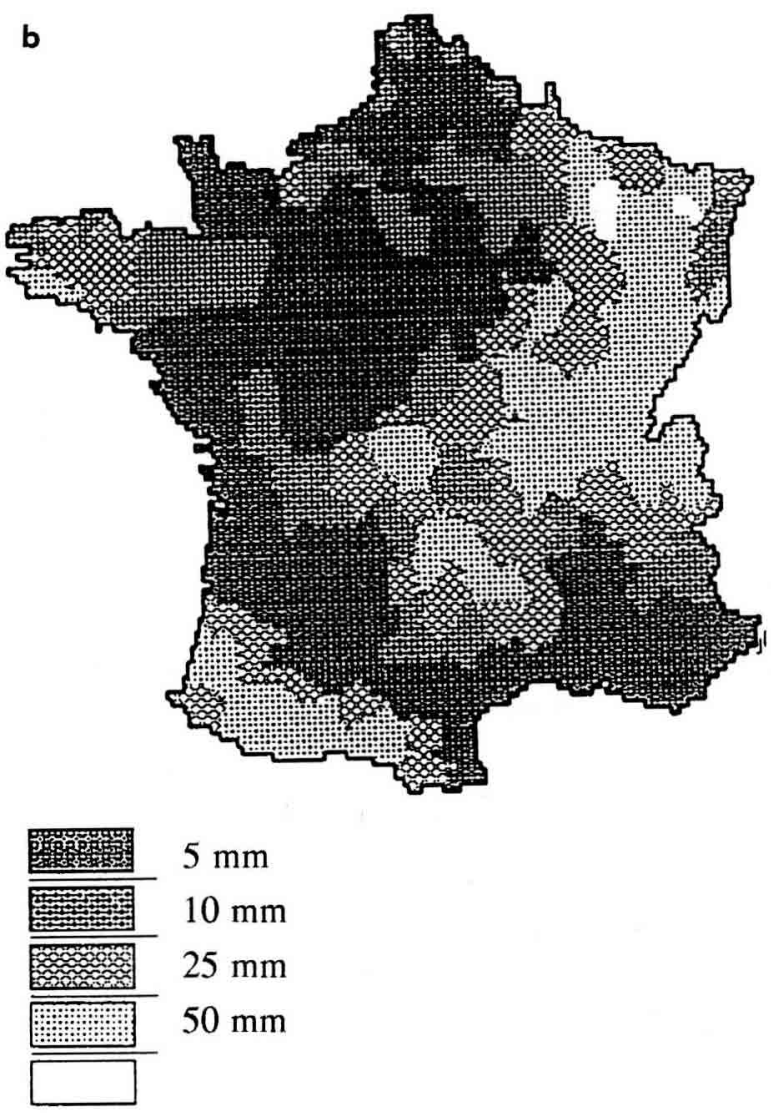

Fig 9b. Carte de la réserve du sol calculée d'après un bilan hydrique à pas de temps mensuel, établi au 31 juillet 1990 à partir de données de la météorologie nationale (extraite de la note du 3-8-1990. Bureau de l'hydraulique agricole et des grands ouvrages). c

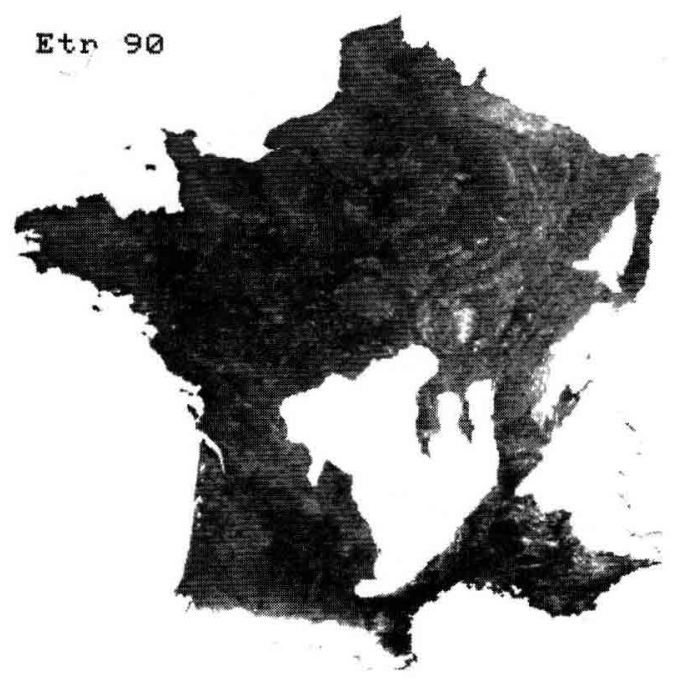

d

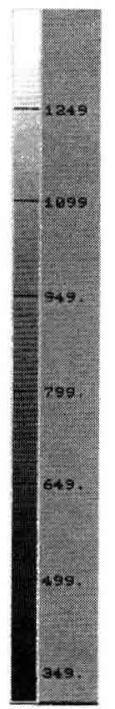

Fig 9c. Carte de l'ETR intégrée de mars à octobre 1990 , obtenue à partir des données NOAA.
Fig 9d. Zoom de la carte de l'ETR en 1990 sur le secteur Sud-Ouest. 
2 estimations d'ETR pour la période étudiée de mars à octobre sont de l'ordre de $\pm 7 \mathrm{~mm}$.

\section{Extrapolation à l'ensemble de la France, détection des effets de sécheresse}

Des images résultant du cumul des températures de surface obtenues à partir des 48 images AVHRR ont été réalisées pour chacune des 3 années étudiées. L'année 1988 apparaît plus froide sur l'ensemble de la France par rapport aux années 1989 et 1990 (fig 8 ). Ceci correspond bien aux observations météorologiques faites sur les 20 stations (tableau I). Les secteurs, où les différences entre années sèches et humides sont les plus marquées, se situent surtout à l'ouest de la France (Sud-Ouest, Charente, Normandie). On observe peu d'écart entre les années 1989 et 1990 , les températures de surface étant légèrement plus élevées en 1989 dans les régions centre qu'en 1990.

Les cartes des variables climatiques (Tair, $\mathrm{Rn}$ ) obtenues par krigeage ont été combinées aux images AVHRR de NOAA suivant la relation simplifiée en utilisant les coefficients de référence calculés à partir des 20 stations. Des masques ont été appliqués sur les reliefs car nous ne possédions pas de données climatiques sur ces secteurs (fig 9c).

Ces images ont été comparées à des cartes (utilisant des moyennes départementales, et des données fournies par la météorologie nationale sur 183 postes) ainsi qu'à des images de synthèse basées sur les cumuls de l'indice de végétation (cartes élaborées par Geosys, pour détecter les effets de sécheresse à l'échelle régionale (fig 9a, b). La comparaison est satisfaisante, puisque l'on retrouve les mêmes secteurs les plus touchés par la sécheresse. Les valeurs d'évaporation estimées montrent des différences importantes, de plus de $100 \mathrm{~mm}$, entre l'année 1988 et les années 1989-1990 pour certains secteurs comme le sud-ouest ou la Charente-Maritime (on relève les valeurs suivantes d'évaporation de mars à octobre pour SaintLaurent-de-la-Prée: ETR88 $=503 \mathrm{~mm}$, ETR89 = $356 \mathrm{~mm}, E T R 90=317 \mathrm{~mm}$ ). Ces résultats sont en concordance avec les données météorologiques (Guiméra, 1992) qui expriment un déficit de l'ordre de $200 \mathrm{~mm}$ en 1990 pour le sud-ouest par rapport à la normale. Ces cartes permettent une évaluation globale de la sécheresse à l'échelle d'un cycle de végétation. En considérant des pas de temps plus courts, la même méthodologie pourrait être utilisée pour effectuer un suivi temporel des conditions hydriques des cultures et permettre d'identifier les phases de fort déficit hydrique. La température de surface apparaît ainsi complémentaire de l'information donnée par l'indice de végétation. Elle présente de plus l'avantage de traduire de façon instantanée les conditions hydriques de la surface, alors que l'indice de végétation ne réagit aux stress subis par les cultures qu'après plusieurs jours, son interprétation pouvant être aussi plus délicate.

\section{CONCLUSION}

Cette étude a montré l'intérêt des données satellitaires thermiques permettant une estimation globale de l'état hydrique des cultures à large échelle. Elle a permis de confirmer la localisation des régions les plus touchées par les sécheresses en 1989 et 1990: le SudOuest, la Charente, la Normandie. L'examen visuel des différentes cartes issues des données thermiques montre que la méthode proposée présente une concordance apparente avec celle utilisant l'indice de végétation.

Un certain nombre de problèmes demeurent cependant, notamment au niveau de la chaîne de traitement des images où des corrections atmosphériques seraient nécessaires afin d'améliorer la précision des températures de surface. La cartographie de l'occupation de la surface serait également utile. Des travaux récents (Derrien et al, 1992) fournissent des résultats préliminaires de classification de l'occupation de surface obtenues à l'échelle de la France à partir de l'indice de végétation calculée sur des images AVHRR des satellites NOAA. Ces classifications pourraient être utilisées à l'avenir pour en sortir des cartes dérivées des paramètres de surface comme la rugosité de surface, l'albedo ou l'émissivité, intervenant dans les modèles agrométéorologiques, et améliorer ainsi la précision des estimations régionales d'évapotranspiration.

\section{REMERCIEMENTS}

Nous tenons à remercier particulièrement MC Imberti (Geosys, Toulouse) qui nous a fourni des in- 
formations concernant les cartographies régionales, $\mathrm{P}$ Monestiez et $\mathrm{T}$ Goulard (Biométrie, INRA Montfavet) pour leurs conseils au cours de ce travail concernant la spatialisation des données climatiques.

\section{RÉFÉRENCES}

Becker F, Li ZL (1990) Towards a local split-window method over land surfaces. Int $J$ Remote Sens $11,3,369-393$

Berges JC, Cunin L, Rigal D (1989) Évolution de la végétation sur zone sahélienne. Veille sate/litaire, ORSTOM, Lannion, 36

Brutsaert W (1975) On a derivable formula for long-wave radiation from clear skies. Water $R e-$ sour Res 11, 742-744

Bureau de l'hydraulique et des grands ouvrages (1990) "Situation agro-hydro-météorologique fin juillet 1990 ", note du 3-8-1990

Carlson TN, Buffum MJ (1989) On estimating total evapotranspiration from remote surface temperature measurements. Remote Sens Environ $29,197-207$

Chanzy A (1991) Modélisation simplifiée de l'évapotranspiration d'un sol nu utilisant l'humidité et la température de surface accessibles par télédétection. Thèse I'INAPG-INRA Avignon, Montfavet, $221 \mathrm{p}$

Choisnel E (1977) Le bilan d'énergie et le bilan hydrique du sol. La météorologie, numéro spécial “Évapotranspiration ", VI, 11, 103-133

Derrien M, Farki B, Le Gléau H, Sairouni A (1992) Vegetation cover mapping over France using NOAA-11/AVHRR. Int $J$ Remote Sens 697554B, $1-9$

Deschamps PY, Phulpin T (1980) Atmospheric correction of infrared measurements of sea surface temperature using channels at $3.7,11$ and $12 \mu \mathrm{m}$. Boundary Layer Meteorol 18, 131-143

Gate P, Bouthier A, Moynier A (1992) La tolérance des variétés à la sécheresse: une réalité à valoriser. Perspectives agricoles, $n^{\circ} 169,62-67$

Geosys (1990) Mise en place d'un système de veille sur l'état de la végétation en quasi-temps réel. Rapport ISPRA, contrat $n^{0} 40399007$ ED ISP F

Girard G, Ledoux E, Villeneuve JP (1981) Le modèle couplé: simulation conjointe des écoulements de surface et des écoulements souterrains sur un système hydrologique. Cahier de l'ORSTOM, "série hydrologique", 18, 4, 195-279

Guérif $M$, Delecolle R, Gu X, Guinot JP, Jappiot $M$, Steinmetz $S$ (1988) Estimation de la biomasse et du rendement de cultures de blé à partir des indices de végétation SPOT. Proc 4th Int Coll on "Spectral Signatures of objects in Remote Sensing ", Aussois, ESA SP 287, 137-141
Guiméra M (1992) La sécheresse en France: 1988-1991. Atmosphere, climat. Lettre d'information météorol nat, $\mathrm{n}^{\circ} 27,14$

Itier B, Riou C (1982) Une nouvelle méthode de détermination de l'évaporation réelle par thermographie IR. J Rech Atmos 16, 113-125

Jackson RD, Reginato RJ, Idso SB (1977) Wheat canopy temperature: a pratical tool for evaluating water requirement. Water Resour Res 13, 3, 651-65

Justice CO, Hiernaux PHY (1986) Monitoring the grasslands of the Sahel using NOAA AVHRR data: Noger 1983. Int J Remote Sens 7, 14751497

Kanemasu ET, Verma SB, Smith EA et al (1992) Surface flux measurements in FIFE: an overview. J Geophys Res 97, nº17, 18, 547-555

Kerr $Y$ (1991) Corrections atmosphériques dans l'infrarouge thermique. Cas de l'AVHRR. $5^{e}$ Coll Int "Mesures physiques et signatures en télédétection". Courchevel, ESA SP 319, 29-34

Kneizys FX (1983) Atmospheric transmittance/radiance: computer code Lowtran 6, Technical report AFGL-TR-83-0187, Optical Physics Division, US AIR Force geophysics laboratory, Hanscom air force base, MA USA.

Lagouarde JP, Brunet $Y$ (1991) Suivi de l'évapotranspiration réelle journalière à partir des données NOAA-AVHRR lors de la campagne Hapex-Mobilhy. $5^{\mathrm{e}}$ COLL INT "Mesures physiques et signatures en télédétection". Courchevel, ESA SP 319, 569-572

Lagouarde JP, MacAneney KJ (1992) Daily sensible heat flux estimation from a single measurement of surface temperature and maximum air temperature. Boundary-Layer Meteorol 59, 4, 341-362

Météo-France (1989-1990) Résumés climatologiques de 1989 à 1991. La météorologie, $7^{\mathrm{e}}$ série, $28,29,30,31$

Ottlé C, Vidal-Madjar D (1992) Estimation of land surface temperature with NOAA9 data. Remote Sens Environ 49, 1, 27-41

Perrin JL (1991) Modélisation mathématique du fonctionnement hydrologique des bassins versants de la Fecht et de la Weiss (Haut-Rhin). Thèse $3^{\mathrm{e}}$ cycle univ Louis-Pasteur, Strasbourg I, $212 p$

Price JC (1983) Estimating surface temperature from satellite thermal infrared data. A simple formulation for the atmospheric effect. Remote Sens Environ 13, 353-361

Price JC (1991) Timing of NOAA afternoon passes. Int J Remote Sens 12, 1, 193-198

Riou C, Itier B, Seguin B (1988) The influence of surface roughness on the simplified relationship 
between daily evaporation and surface temperature. Int J Remote Sens 9, 1529-1533

Seguin B, Baelz S, Monget JM, Petit V (1982) Utilisation de la thermographie IR pour l'estimation de l'évaporation régionale. II. Résultats obtenus à partir de données satellites. agronomie 2, 113118

Seguin B, Itier B (1983) Using midday surface temperature to estimate daily evaporation from satellite thermal IR data. Int J Remote Sens 4, 2, 371-383

Seguin B, Assad E, Freteaud JP, Imbernon J, Kerr $\mathrm{YH}$, Lagouarde JP (1989) Use of meteorological satellite for water balance monitoring in sahelian regions. Int J Remote Sens 10, 1001-1017

Seguin B, Lagouarde JP, Savane M (1991) The assessment of regional crop water conditions from meteorological satellite thermal infrared data. Remote Sens Environ 35, 141-148

Seguin B (1992) NOAA-AVHRR data for crop monitoring at a regional level: possibilities and limits in the European context. Int: Symposium EARSEL sur les applications de la télédétection à l'agrométéorologie. Florence, Italie, 13-14 avril 1992. Advances in Remote Sensing 2, 87-93

Soer GJ (1980) Estimation of regional evapotranspiration and soil moisture conditions using surface temperatures. Remote Sens Environ 9, 27-45

Steinmetz S, Lagouarde JP, Delecolle R, Guérif $M$, Seguin B (1989) Evaporation and water stress using thermal infrared measurements. A general review and a case study on winter durum wheat in southern France. Symposium on physiology breading of winter cereals for stressed Mediterranean environments. ICARDA-INRA, juillet 89 , Montpellier, INRA 55, 89-114

Stoll MP (1988) Mesures de la température et de l'émissivité de surface par télédétection: modèles et méthodes. Télédétection spatiale, aspects physiques et modélisation, CNES, école d'été, 845-904

Taconet O, Bernard R, Vidal-Madjar D (1986) Evapotranspiration over agricultural region using a surface flux/temperature model based on NOAA-AVHRR data. J Clim Appl Meteorol 25, 284-307

Turc L (1984) Le bilan d'eau des sols, relations entre les précipitations, l'évaporation et l'écoulement. Ann Agron 4, 491-595

Tucker CJ, Gatlin JA, Schneider SR (1984) Monitoring vegetation in the Nile delta with NOAA6 and NOAA7 AVHRR imagery. Phot Eng Remote Sens 50, 53-62

Tucker CJ (1979) Red and photographic infrared linear combinations for monitoring vegetation. Remote Sens Environ 8, 127-150

Van de Griend AA, Owe M, Stoll MP (1991) Measurement and spatial variation of thermal infrared surface emissivity in a Savanna environment. Water Resour Res 27, 3, 371-379

Vidal A, Kerr Y, Lagouarde JP, Seguin B (1987) Télédétection et bilan hydrique : utilisation combinée d'un modèle agrométéorologique et des données de I'IR thermique du satellite NOAAAVHRR. Agric For Meteorol 39, 155-175 